\title{
DERECHOS DE PROPIEDAD INTELECTUAL (DPI) Y CRECIMIENTO ECONÓMICO: UNA REVISIÓN
}

\author{
RICARDO ALONSO TEJEDOR ESTUPIÑÁN*, JOSÉ MAURICIO GIL LEÓN*** \\ \& JOAN MIGUEL TEJEDOR ESTUPIÑÁN**** \\ UNIVERSIDAD PEDAGÓGICA Y TECNOLÓGICA DE COLOMBIA- UPTC.
}

Recibido/ Received/ Recebido: 08/09/2016 - Aceptado/ Accepted / Aprovado: 30/09/2016

\begin{abstract}
Resumen
Este artículo analiza los efectos de los Derechos de Propiedad Intelectual (DPI) sobre el crecimiento económico de los países, donde una mayor protección incentiva procesos de innovación que inciden sobre el mayor avance de la producción. En el desarrollo analítico del trabajo se toma como marco teórico el modelo de crecimiento endógeno de Romer (1991), el cual explica los fundamentos de cómo la formación de conocimiento conduce a que las economías eleven sus niveles de desarrollo. Para dar sustento a lo expuesto, se encuentra en la literatura, diferentes trabajos en los que se ha comprobado la importancia de los PDI. Finalmente, tomando una muestra de países entre 1990 y 2014 se concluye que el gasto en formación de conocimiento complementado con mayor protección en DPI conduce a que exista un mayor crecimiento económico per cápita, pero lo fundamental resulta ser el gasto en I+D y no el capital humano definido como el porcentaje de la población con educación superior.
\end{abstract}

Palabras clave: Derechos; Propiedad intelectual; Crecimiento económico; Investigación y desarrollo.

\section{INTELLECTUAL PROPERTY RIGHTS (IPR) AND ECONOMIC GROWTH: A REVIEW}

\begin{abstract}
This article analyzes the effects of Intellectual Property Rights (IPR) on the economic growth of countries, where greater protection encourages innovation processes that affect the greater advance of production. In the analytical development of work, Romer's (1991) endogenous growth model is taken as a theoretical framework, which explains the foundations of how knowledge formation leads economies to raise their levels of development. To give support to the above, is found in the literature, different works in which the importance of the IPR has been proven. Finally, taking a sample of countries between 1990 and 2014, it is concluded that spending on knowledge formation complemented by greater IPR protection leads to greater economic growth
\end{abstract}

Magister en Economía. Investigador Grupo Modeal Universidad Pedagógica y Tecnológica de Colombia- UPTC, Colombia. ORCID ID: orcid.org/0000-0002-5877-846X. Dirección postal: Av. Central del Norte, UPTC, Tunja, Edificio Central, Oficina C-342a. Teléfono: +57 3214609895. Correo Electrónico: ricardotse@hotmail.com

* Economista, especialista en Finanzas y Magister en Economía. Docente de teoría económica e Investigador Grupo Oikos Universidad Pedagógica y Tecnológica de Colombia- UPTC, Colombia. Correo electrónico: mauricio8827@hotmail.com

Magíster en Derechos Humanos y economista. Universidad Católica de Colombia. ORCID ID: orcid.org/0000-0002-2346-3222. Correo electrónico: jmtejedor@ucatolica.edu.co 
per capita, but the main thing turns out to be spending on $\mathrm{R} \& \mathrm{D}$ and not human capital defined as the percentage of the population with higher education.

Keywords: Rights; Intellectual property; Economic growth; Research and development.

\title{
DIREITOS DE PROPRIEDADE INTELECTUAL (DPI) E CRESCI- MENTO ECONÔMICO: UMA REVISÃO
}

\begin{abstract}
Resumo
Este artigo analisa os efeitos dos Direitos de Propriedade Intelectual (DPI) sobre o crescimento econômico dos países, onde uma maior proteção incentiva processos de inovação que incidem sobre o maior avanço da produção. No desenvolvimento analítico do trabalho toma-se como marco teórico o modelo de crescimento endógeno de Romer (1991), o qual explica os fundamentos de como a formação de conhecimento conduz a que as economias elevem seus níveis de desenvolvimento. Para dar sustento ao exposto, encontram-se na literatura, diferentes trabalhos nos que se comprovou a importância dos DPI. Finalmente, tomando uma mostra de países entre 1990 e 2014 conclui-se que a despesa em formação de conhecimento complementado com maior proteção em DPI conduz a que exista um maior crescimento econômico per capita, mas o fundamental resulta ser a despesa em I+D e não o capital humano definido como a percentagem da população com educação superior.

Palavras chave: Direitos; Propriedade intelectual; Crescimento econômico; iNvestigação e desenvolvimento.

Tejedor, R., Gil, J. \& Tejedor, J. (2018). Derechos de Propiedad Intelectual (DPI) y crecimiento económico: una revisión. En: Revista de la Facultad de Ciencias Económica: Investigación y Reflexión. rev.fac.cienc.econ, XXVI (1), DOI: https://doi.org/10.18359/rfce.3145
\end{abstract}

JEL: O34, O47, O32.

\section{Introducción}

En este trabajo se explora la influencia que tienen los Derechos de Propiedad Intelectual (DPI) sobre el comportamiento del crecimiento económico en los diferentes países seleccionados, y para ello se tendrá como referente el modelo planteado por Gould \& Gruben (1996), el cual evidencia la relación entre la protección a los derechos de propiedad intelectual y sus efectos sobre los procesos de innovación y progreso tecnológico, elementos que han sido abordados para explicar el crecimiento económico en economías abiertas.

Los DPI al igual que los derechos a la propiedad privada, son el conjunto de instrumentos legales que surgieron el desarrollo mismo de las civilizaciones, y se establecieron para garantizar la protección de la creación del intelecto humano, como las invenciones, las obras literarias y artísticas, etc., y con el propósito de otorgar al titular el derecho, el uso exclusivo del producto de su intelecto y plasmado en la obra, el descubrimiento o la innovación, etc., y así obtener los beneficios del mismo. Los DPI tienen una legislación específica y su aplicación depende de los acuerdos establecidos principalmente en procesos de integración económica. Por lo anterior, los DPI han ido modificándose a medida que se han ido fortaleciendo, principalmente por los países cuya producción de bienes para exportación dependen del desarrollo científico, dadas las ventajas competitivas que ello puede representar en el comercio mundial. 
La explicación más influyente de cómo el avance tecnológico se involucra en el crecimiento económico es dada por la teoría del crecimiento endógeno (finales de la década de los ochenta del siglo XX), y desde entonces se difunde el estudio teórico y empírico sobre la forma intrínseca de incluir variables como capital humano, conocimiento, innovación, gasto público en educación, inversión en $\mathrm{I}+\mathrm{D}$, con el objeto de reducir costes, generar nuevos procesos y obtener mejores productos. No obstante, el origen teórico se encuentran principalmente en los aportes de Allyn Young (1928), quién ofrece una visión moderna de la tradición Smithsoniana de la división del trabajo; esta idea fue desarrollada por Romer (1983) en su disertación doctoral: Dynamic competitive equilibria with externalities, increasing returns and unbounded growth (Equilibrios competitivos dinámicos con externalidades, rendimientos crecientes y el crecimiento sin límites) y su posterior artículo de 1987: Growth based on increasing returns due to specialization (El crecimiento basado en el aumento de los rendimientos debido a la especialización).

Romer (1987) propone el conocimiento como la variable que permite explicar el cambio técnico, $y$, de esta forma, aumentar la productividad de los factores. En ese sentido, se señala que el conocimiento es generador de mejoras en los productos dentro y entre industrias, donde las externalidades relacionadas con su uso, constituyen un determinante fundamental en el marco de la teoría de crecimiento endógeno (Guzmán, 2000). Además, es de anotar que el progreso tecnológico es un elemento generado por la actividad $e$ interés de los agentes económicos involucrados directa o indirectamente en el proceso de innovación, investigación o diseño de nuevos procesos o productos; y, por esta razón, la corriente del crecimiento endógeno propone canales por los cuales la tasa de progreso tecnológico y la tasa de crecimiento económico a largo plazo pueden ser influenciados por el tipo de gobierno:

Según Sala I Martin (2000), "En este sentido es deseable la aparición de gobiernos que garanticen los derechos de propiedad física e intelectual, que regulen el sector financiero y exterior y eliminen las distorsiones, y que mantengan un marco legal garante del orden" (p. 6).

Los primeros modelos planteados por Romer (1983, 1987), Lucas (1988) y Rebelo (1991) no incluyeron el cambio tecnológico como una variable inherente, en dichos modelos prevaleció la idea de que el crecimiento puede continuar de forma indefinida, ya que los rendimientos provenientes de la inversión en capital humano no disminuyen, y esto se debe a que la difusión del conocimiento entre productores y las externalidades positivas del capital humano forman parte de este proceso (Barro \& i Martin, 2009: 19). Los modelos de crecimiento endógeno integran los conceptos de externalidades positivas, aprendizaje y capital humano para concebir la posibilidad de un progreso técnico desde dentro del sistema económico.

Las fuentes del progreso técnico que permiten el crecimiento de la riqueza deben ser buscadas entonces en la producción -más allá incluso del capital y del trabajo-y fuera del mercado, resolviendo el problema de los rendimientos decrecientes que impone la hipótesis de la competencia pura y perfecta y la teoría de la redistribución basada en la productividad marginal de los factores (Corsani, 2004: 94). Para Benavides et al. (2002), la tradición de Romer (1991) debe asegurar una condición monopólica, ya que de lo contrario en competencia perfecta no puede haber equilibrio, mientras que para Lucas (1988) hay competencia perfecta.

De acuerdo a lo expuesto teóricamente, se examina el papel que cumplen los DPI en el crecimiento económico, utilizando información de corte transversal para 66 países en el periodo 1990-2014, donde las variables incluidas son: Formación Bruta de Capital como proporción del PIB, población activa con educación terciaria, gasto en I + D respecto del PIB, el grado de apertura comercial, PIB per cápita, gasto público como proporción del PIB, nivel de renta de los países, y la protección de los DPI a través del indice IPRI. Los resultados indican que la protección de los DPI -medida a través del índice de protección de propiedad intelectual IPRI- está relacionada con el crecimiento económico. 
Una pregunta inconclusa que subyace sobre el tema de la protección al conocimiento o a la propiedad del intelecto, es la siguiente: ¿Cuál es el nivel adecuado de propiedad sobre el conocimiento que permite la apropiación de los beneficios sociales y privados del mismo y por tanto el crecimiento económico? Entonces, la discusión en torno a la protección de los derechos de propiedad intelectual en el marco de la nueva economía del conocimiento, implica encontrar una medida optima de protección de los DPI, que permita tanto la apropiación privada de las ganancias como la difusión del conocimiento y la apropiación social de las innovaciones, mejoras o avances de la técnica, teniendo en cuenta las diferencias sustanciales en las estructuras políticas, económicas y sociales de los países.

Para cumplir el propósito de realizar una aproximación al conocimiento, el crecimiento económico y sus relaciones con los DPI, el trabajo se desarrolla en cinco secciones, tomando la presente como la primera. En la segunda sección se analiza el modelo del crecimiento endógeno de Romer (1991) como el marco de teórico del documento. La tercera sección aborda algunas generalidades sobre la relación entre la dureza en la aplicación de los DPI, el cambio técnico y el crecimiento económico, tomando como punto de referencia el trabajo de Gould \& Gruben (1996), lo que permite entender cuál es la importancia que tienen los DPI para el crecimiento económico entre países de diferente nivel de ingresos, educación, grado de apertura comercial y estructura económica. En la cuarta sección se realiza una comprobación empírica, con una muestra de 66 países entre 1990 y 2014, que permite estimar el efecto de la protección intelectual sobre el crecimiento económico per cápita de los países. Finalmente, se formulan algunas conclusiones sobre las discusiones y los resultados mostrados en el documento.

\section{Teoría del Crecimiento Endógeno}

En su trabajo de 1991, Romer analiza porqué los economistas neoclásicos notaban indirectamente que el producto por hora trabajada se incrementaba debido al cambio tecnológico y al papel que juegan el descubrimiento de nuevos métodos para transformar las mismas materias primas y cómo debido a esto los ingresos de los trabajadores se había incrementado; para poder explicar lo anterior Romer se basó en tres premisas:

- El cambio tecnológico aporta el incentivo necesario para la acumulación continua de capital; y la acumulación de capital y el cambio tecnológico en su conjunto son responsables de gran parte del incremento del producto por hora trabajada.

- El modelo es de cambio tecnológico endógeno más bien que de exógeno, ya que este surge de las acciones intencionales realizadas por personas que responden a los incentivos del mercado.

- Las instrucciones para trabajar con las materias primas son inherentemente distintas de otros bienes económicos, por lo que esto implica que la tecnología es un insumo no rival. Una vez creado un nuevo conjunto de instrucciones, éstas pueden usarse una y otra vez sin ningún costo adicional.

Los factores de producción considerados son: El capital $K$, la mano de obra $L$, el capital humano $H$ (es un bien rival), y el componente tecnológico no rival $A$. El modelo separa al componente rival del conocimiento $H$, del componente no rival, del conocimiento aplicado a un diseño tecnológico $A$, el cual puede crecer ilimitadamente, lo cual ocurre únicamente en ausencia de derechos de propiedad para el uso de los nuevos conocimientos dentro del proceso de producción. Es de aclarar que la rivalidad es un atributo puramente tecnológico. Un bien puramente rival tiene la propiedad de que su uso por una empresa o persona impide su uso por otra; un bien puramente no rival tiene la propiedad de que su uso por una empresa o persona no limita en modo alguno su uso por otra (Romer, 1991: 445). La posibilidad de exclusión es una función de la tecnología y del sistema legal. Un bien es susceptible de exclusión si el propietario puede impedir que otros lo usen. Un bien tal como el código para un programa de computadora puede hacerse excluible mediante un sistema legal que prohíba la copia, o mediante programas de protección de la grabación y de las copias (Romer, 1991: 445). 
Los bienes económicos tradicionales son a la vez rivales y excluibles. Se proporcionan privadamente $y$ pueden comerciarse en mercados competitivos; por su parte, los bienes públicos son no rivales y no excluibles al mismo tiempo. No pueden proporcionarse de manera privada ni comerciarse en los mercados. Pueden introducirse en un modelo de conducta de toma de precios suponiendo la existencia de un gobierno que puede cobrar impuestos. El conocimiento es asumido, entonces, como un bien público por su condición de no rivalidad y no exclusión, debido a esto aparecieron los DPI como un instrumento legal que propiciará la apropiación del mismo, ya que de lo contrario serían pocos los incentivos.

Según un informe del CECTE ${ }^{1}$ (2008: 4) “(...) Los gobiernos encontraron diversos modos de tratar este problema. Uno consistió en financiar la producción y entrega de bienes públicos de manera directa o conceder fondos o subsidios a terceros para que produzcan o provean dichos bienes. Otra, fue otorgar derechos de propiedad a los productores de bienes públicos a fin de permitirles ejercer cierto control sobre el uso de sus creaciones. En este sentido, los DPI fueron concebidos como una manera de tratar la apropiación de bienes públicos mediante la concesión a los creadores de derechos exclusivos sobre el uso de sus obras durante un tiempo determinado".

Por otra parte, la rivalidad y la posibilidad de exclusión están ligadas estrechamente porque la mayoría de los bienes rivales son excluibles (Romer, 1991: 445). Para la teoría del crecimiento económico de Romer (1991) los bienes a analizar son aquellos que no son rivales y sin embargo son excluibles. Entonces, la primera de las premisas señaladas implica que el crecimiento es impulsado fundamentalmente por la acumulación de un insumo parcialmente excluible, no rival (Romer, 1991: 445) y este insumo es el conocimiento. En ese sentido, debido a los atributos característicos de los bienes públicos, como el caso del conocimiento y las ideas innova-

$\overline{1}$ Comité Nacional de Ética en la Ciencia y la Tecnología de Argentina. doras, los agentes querrán aprovechar la reducción de costos que implica su uso, lo anterior gracias a su condición de no rivalidad, pero la posibilidad de que se de exclusión, dependerá de la aplicación de un régimen de DPI.

El modelo de Romer (1991) tiene tres sectores que conforman la economía: el primero es el sector de la investigación basado en el acervo de conocimiento y el capital humano existente para producir nuevo conocimiento a través de nuevos diseños y bienes de producción duraderos; el segundo produce bienes intermedios usando los diseños $y$ conocimiento del primero junto con el producto sacrificado para elaborar bienes de producción duraderos disponibles para producción de bienes finales, la investigación y posterior diseño o invento puede ocurrir internamente o comprarse a través de una patente de un segundo; el tercero, es el sector de bienes finales el cual utiliza mano de obra, capital humano y el conjunto de bienes de producción duraderos que están disponibles para generar el producto final, el cual puede consumirse o ahorrarse como nuevo capital (Romer, 1991).

En el modelo se asume: a) La población y la oferta de mano de obra son constantes; b) El acervo total de capital humano en la población está fijo, la oferta de los factores agregados $L$ y $H$ está fija; c) Existe un desplazamiento de recursos del sector de consumo al sector de capital ${ }^{2}$. El producto final corresponde a $Y$, el cual está en función de la mano de obra física $L$, el capital humano $H y$ y el capital físico $x$. La tecnología de producción desagrega el capital en un número infinito de tipos diferentes de bienes duraderos que se indican por un entero $i .^{3}$

Sólo los insumos potenciales -los que se ya se han inventado y diseñado- están disponibles para usarse, $x=\left\{x_{i}\right\}_{i=1}^{\infty}$ entonces es la lista de los insumos usados por una empresa que obtiene un producto

\footnotetext{
Se afirma que la investigación es relativamente intensiva en capital humano y conocimientos para producir nuevos diseños o conocimientos.

3 Romer (1991) supondrá que el índice es una variable continua, no discreta, con el fin de eludir restricciones por el uso de valores enteros.
} 
final. Entonces, cambia a medida que se inventan nuevos bienes duraderos. El modelo considera la función de producción tradicional:

$$
Y\left(H_{y}, L, x\right)=H_{\gamma}^{\alpha} L^{\beta} \sum_{i=1}^{\infty} x_{i}^{1-\alpha-\beta}
$$

Romer (1991) define implícitamente el capital K como proporcional a la suma de todos los tipos de bienes de capital diferentes de la economía, los cuales son sustitutos perfectos, cercanos, pares complementarios y pares intermedios. Por otro lado, al sumar a todas las personas ocupadas en investigación, el acervo agregado de los diseños evolucionará de acuerdo con la ecuación [2], donde $H_{A}$ es el capital humano total empleado en la investigación:

$$
\dot{A}=\delta H_{A} A
$$

Todos los investigadores pueden aprovecharse de $A$ al mismo tiempo. Por lo tanto, el producto del investigador j es $\delta H_{A} A$. Romer (1991) explica que el nuevo conocimiento es apropiable libremente en el proceso de producción porque, al ser copiados los nuevos diseños se permiten fabricar productos nuevos, lo cual aumenta el acervo de conocimiento $e$ incrementa la productividad del capital humano en el sector de investigación, pero precisamente el conflicto surge cuando un nuevo diseño es copiado. El efecto de que tiene el hecho de no poseer derechos de propiedad sobre el conocimiento, hace que su acceso por parte del sector de I+D genere mayor crecimiento en $A$ y, por ello, en la productividad del capital humano.

Romer (1991) concluye que en un escenario de libre comercio, una mayor acumulación de capital humano, permite acelerar el crecimiento económico, dado que este parece estar correlacionado con el grado de integración a los mercados mundiales y no con el tamaño o la densidad de la población. Con respecto al papel del conocimiento argumenta que los proyectos de investigación incentivan los beneficios futuros. Por tanto, la tasa de cambio tecnológico es sensible a la tasa de interés.
La acumulación de capital físico es un sustituto muy deficiente en relación con el incentivo para emprender la investigación. En ausencia de políticas viables que puedan eliminar la divergencia entre los rendimientos sociales y los privados de la investigación, una política de subóptimo sería el subsidio a la acumulación de capital humano total (Romer, 1991: 476). Un artículo posterior Romer (1994) vendría a analizar los orígenes del crecimiento endógeno, en éste trata de desentrañar dos elementos; la controversia de la convergencia, y la discusión entre la aplicabilidad de la teoría económica a un nivel agregado en el marco de la competencia perfecta.

\section{Relaciones entre los DPI y el crecimiento económico}

Existen estudios empíricos que han buscado evaluar el efecto del nivel de protección con DPI en el crecimiento económico, aunque dichos enfoques tienen la ventaja de señalar un efecto importante entre dichas variables, ha tenido algunos inconvenientes, ya que el efecto de los DPI sobre el crecimiento no ha sido claro. Uno de los primeros en examinar el efecto de protección de los DPI sobre el crecimiento económico así como frente a los acuerdos de integración emergentes conocidos como: Trade-Related Aspects of Intellectual Property Rights o TRIPS fue Scott Taylor (1994), el cual presenta un modelo donde vincula el régimen de DPI con el régimen comercial, el nivel agregado de I + D, la transferencia de tecnología y el crecimiento económico. Según Taylor (1994), el no proporcionar protección de patentes a las innovaciones extranjeras lleva a los innovadores locales a emplear menos de las mejores tecnologías de investigación, reduce las actividades globales de investigación y desarrollo, elimina de manera efectiva la transferencia de tecnología entre los países, y reduce el crecimiento.

Posteriormente, Gould \& Gruben (1996) formulan un modelo para examinar la relación que tiene la protección de los DPI en el crecimiento económico, en un régimen de comercio abierto; demuestran que la protección a la propiedad intelectual podría estimular la innovación y, por lo tanto, el crecimiento económico, aunque, sugieren que la innovación 
puede desempeñar un papel menos relevante en países con mercados menos competitivos. Sugieren que una liberalización del comercio, acompañada de una fuerte protección a la propiedad intelectual, es un importante conductor para el crecimiento económico.

El modelo de Gould \& Gruben (1996) utiliza datos de corte transversal para diferentes países, utilizando variables como la protección de patentes, medida por el indice (IPROP) de Rapp \& Rozek (1990) ${ }^{4}$ $y$ otras variables de control. La proxy se basa en las leyes de patentes de cada nación respecto a los estándares mínimos propuestos por las instituciones concernientes. El índice ordena el nivel de protección de patentes en una escala del 1 al 6 , donde 1 corresponde a las naciones que no tiene ninguna ley para la protección de patentes y 6 a aquellas cuyas leyes son totalmente congruentes con los estándares mínimos (Gould \& Gruben, 1996).

Los autores recurren a regresiones utilizando el enfoque de variable instrumental, con el fin de tener en cuenta los posibles errores de medición y endogeneidad. Gould \& Gruben (1996) emplean tres medidas para capturar la apertura de un país: la prima de mercado negro (BMP), la distorsión del tipo de cambio real (RERMED), y un índice de comercio. Para las tres medidas de apertura se encuentran que el impacto positivo significativo de IPROP es más fuerte en relación con las economías más abiertas.

Otro estudio similar es el de Ginarte \& Park (1997), quienes construyen un índice ${ }^{5}$ para mirar la relación

Para elaborar su índice, Rapp \& Rozek (1990) se basaron en Gadbaw \& Richards (1988), para quienes el alcance de la protección de patentes y el valor del indice resultante se basa en las leyes existentes en contra del infringimiento de las patentes y no en la aplicación de las mismas (Gould \& Gruben 1996).

5 El indice suma cinco puntuaciones separadas para: cobertura (invenciones que son patentables); membresía en los tratados internacionales; duración de la protección; mecanismos de aplicación; y restricciones (por ejemplo, las licencias obligatorias en caso de que una invención patentada no está suficientemente explotada). Este índice fue diseñado para proporcionar un indicador de la fuerza de protección de la patente, no la calidad de los sistemas de patentes (Park, 2008: 761). entre DPI y el crecimiento económico, encuentran que los DPI están relacionados indirectamente con el crecimiento mediante el estímulo a la acumulación de factores de producción como la I + D y el capital físico. Ginarte \& Park (1997) encuentran que los niveles de protección patentes están relacionados con el nivel de actividad en I + D en un país, por lo tanto sugieren crear incentivos y elevar los niveles de protección de patentes en los países que tienen una protección débil como elemento importante para fomentar la investigación.

El índice de Park y Ginarte ha sido actualizado desde su concepción en 1990 cada 5 años, encontrando que el valor medio del índice de protección de patentes ha aumentado con el tiempo, lo que refleja la adopción de leyes de patentes más fuertes entre los países sobre todo después de que los $\mathrm{ADPIC}^{6}$ entraran en vigor, así como la introducción de las leyes de patentes en países que carecían anteriormente de los sistemas de patentes (Park, 2008: 762).

Estudios de Yongmin Chen \& Thitima Puttitanun (2005) llevan a cabo un análisis teórico y empírico de los derechos de propiedad intelectual y la innovación para países en desarrollo, utilizando un conjunto de datos de panel que incluye 64 países en desarrollo durante el período 1975-2000. Resaltan que DPI más leves facilitan imitaciones de tecnologías extranjeras, lo que reduce el poder de mercado de las mismas y los beneficios de los consumidores domésticos, pero advierten que un país en desarrollo debe fortalecer sus sistemas de DPI para fomentar las innovaciones de las empresas nacionales.

Chen \& Puttitanun (2005) demuestran que la innovación en un país en desarrollo aumenta con la protección de los derechos de propiedad intelectual, y que los niveles de protección de DPI óptimos de un país dependen de su nivel de desarrollo (habilidad tecnológica) de una manera no monótona, donde primero disminuyen y luego aumentan. La evidencia empírica confirma el impacto positivo de los DPI sobre las innovaciones en los países en desarrollo y

\footnotetext{
Aspectos de los derechos de propiedad intelectual relacionados con el comercio.
} 
la presencia de una relación en forma de U entre los DPI y los niveles de desarrollo económico.

Finalmente, Chen \& Puttitanun (2005) sugieren que puede haber menos incentivos para proteger los DPI en los países con capacidades innovadoras inferiores o menores niveles de desarrollo tecnológico; por eso, a medida que más países en desarrollo reconocen la importancia de fomentar las actividades empresariales (innovadoras) por las empresas nacionales; existen intereses comunes entre los paí- ses desarrollados y en desarrollo que les llevará a ampliar los DPI. Establecen que en el largo plazo, tal vez la mejor manera para los países desarrollados para promover los DPI de los países en desarrollo es ayudar a estos últimos aumentar las actividades innovadoras.

En adelante se ha desarrollado un amplio aporte y estudios para explicar dichas relaciones entre DPI y crecimiento económico, en el siguiente cuadro se tiene una sintesis de la literatura sobre el tema.

Tabla 1. Impacto de los DPI en la innovación y el crecimiento: una síntesis de la literatura teórica

\begin{tabular}{|c|c|c|}
\hline Conceptualización DPI & Autor (fecha) & Efecto Final Neto \\
\hline \multirow{11}{*}{ Patentes } & Scherer (1997); Koléda (2008) & En la innovación: 0 \\
\hline & Tandon (1982); David and Olsen (1992) & En el bienestar: + \\
\hline & Merges and Nelson (1994) & En el progreso tecnológico: - \\
\hline & Taylor (1994) & $\begin{array}{l}\text { En el crecimiento económico: } \\
+ \text { (protección simétrica) y } \\
\text { - (protección asimétrica). }\end{array}$ \\
\hline & Gould y Gruben (1996) & $\begin{array}{l}\text { En el crecimiento económico: + (en economías abiertas) } \\
\text {-(en economías cerradas) }\end{array}$ \\
\hline & $\begin{array}{l}\text { Michel and Nyssen (1998); Goh and Oliver (2002); Iwaisa- } \\
\text { ko and Futagami (2003) }\end{array}$ & En el crecimiento económico: + \\
\hline & Futagami and Iwaisako (2007) & $\begin{array}{l}\text { En el crecimiento económico: + (longitud patente finita) y } \\
\text { - (longitud patente infinita); una estrategia de patentes con } \\
\text { una longitud finita patente es óptima. }\end{array}$ \\
\hline & Naghavi (2007) & $\begin{array}{l}\text { En el bienestar del Sur: + (si atraer la inversión extranjera } \\
\text { en menos industrias intensivas en I + D o si estimulan la } \\
\text { innovación en sectores de alta tecnología). }\end{array}$ \\
\hline & Dinopoulos and Kottaridi (2008) & $\begin{array}{l}\text { En el crecimiento económico y la distribución del ingreso: } \\
+ \text { (si cada país selecciona el nivel de aplicación de paten- } \\
\text { te de manera óptima, con el Norte que tiene un incentivo } \\
\text { para elegir una protección de DPI más fuerte que el del } \\
\text { Sur). }\end{array}$ \\
\hline & Eicher and Garcia- Peñalosa (2008); Chu (2009a) & En el crecimiento económico: 0 \\
\hline & Panagopoulos (2009) & En el crecimiento económico: una relación cóncava. \\
\hline \multirow{3}{*}{$\begin{array}{l}\text { Índice de derechos de } \\
\text { patentes }\end{array}$} & Gadbaw y Richards (1988) & En el crecimiento económico: + \\
\hline & Rapp y Rozek (1990) & En el crecimiento económico: + \\
\hline & Park (2008) Chu (2010) & $\begin{array}{l}\text { En el crecimiento económico: +; en la desigualdad de } \\
\text { ingresos: } 0\end{array}$ \\
\hline
\end{tabular}




\begin{tabular}{|c|c|c|}
\hline Conceptualización DPI & Autor (fecha) & Efecto Final Neto \\
\hline $\begin{array}{l}\text { Longitud y amplitud } \\
\text { de Patentes; marcas; } \\
\text { derechos de autor y } \\
\text { secretos comerciales; } \\
\text { y el grado de } \\
\text { cumplimiento. }\end{array}$ & Kwan and Lai (2003) & $\begin{array}{l}\text { En el crecimiento económico: grado óptimo de protección } \\
\text { de los DPI. }\end{array}$ \\
\hline \multirow{2}{*}{ Derechos de Autor } & $\begin{array}{l}\text { Novos and Waldman } \\
\text { (1984) }\end{array}$ & En el bienestar social: + \\
\hline & $\begin{array}{l}\text { Landes and Posner } \\
\text { (1989) }\end{array}$ & En el bienestar asociado a una obra determinada: - \\
\hline $\begin{array}{l}\text { Longitud y amplitud } \\
\text { de Patentes; política } \\
\text { de derechos de autor }\end{array}$ & Furukawa (2007) & $\begin{array}{l}\text { En el crecimiento económico: - (cuando el impacto de la } \\
\text { experiencia acumulada en la productividad es lo suficien- } \\
\text { temente grande, se sugiere una relación de U invertida). }\end{array}$ \\
\hline \multirow{4}{*}{$\begin{array}{l}\text { Aumento de los } \\
\text { costos de imitación }\end{array}$} & Stryszowski (2006) & $\begin{array}{l}\text { En el crecimiento económico en países tecnológicamente } \\
\text { rezagadas: } 0\end{array}$ \\
\hline & Glass and Saggi (2002); Mondal and Gupta (2008) & En la innovación y en la IED: - \\
\hline & $\begin{array}{l}\text { Mondal and Gupta (2009); Connolly and Valderrama } \\
(2005)\end{array}$ & $\begin{array}{l}\text { En el bienestar: + (tanto en el Norte y en el Sur, aunque la } \\
\text { ganancia de bienestar marginal es mayor en los primeros } \\
\text { que en el segundo) }\end{array}$ \\
\hline & Wu (2010) & En la innovación: + \\
\hline $\begin{array}{l}\text { Aranceles; Aumento } \\
\text { de los costos de la } \\
\text { imitación }\end{array}$ & $\begin{array}{l}\text { Datta and Mohtadi } \\
(2006)\end{array}$ & En el crecimiento económico del Sur: aranceles (-); IPR (-) \\
\hline \multirow{3}{*}{$\begin{array}{l}\text { Intensidad de } \\
\text { imitación }\end{array}$} & $\begin{array}{l}\text { Mondal and Gupta } \\
\text { (2006); Glass and Wu } \\
\text { (2007); Zhou (2009) }\end{array}$ & En la innovación: 0 \\
\hline & Y. Chen y T. Puttitanun (2005) & $\begin{array}{l}\text { DPI débil: + innovación en países en dllo. } \\
\text { DPI }\end{array}$ \\
\hline & Dinopoulos and Segerstrom (2010) & En la innovación: + \\
\hline $\begin{array}{l}\text { Probabilidad de } \\
\text { imitación y retorno de } \\
\text { la innovación. }\end{array}$ & $\begin{array}{l}\text { Horii and Iwaisako } \\
\text { (2007) }\end{array}$ & En el crecimiento económico: 0 \\
\hline Regalías & Saint -Paul (2008) & En el bienestar: + \\
\hline $\mathbf{N} / \mathbf{a}$ & Furukawa (2010) & En la innovación: U invertida \\
\hline
\end{tabular}

*N/a: no aplicable; 0: efecto neto ambiguo o no concluyente; + (-): efecto neto positivo (negativo).

Fuente: Elaborado por Acevedo et al. (2012), traducción libre

Los partidarios de un sistema fuerte de protección de los DPI argumentan, entonces, que se estimula la adquisición y difusión de conocimientos, ya que la información innovadora no estará disponible para otros potenciales competidores, así los DPI fuertes juegan un papel importante dentro del proceso de apropiación de beneficios para las empresas más competitivas. Si la innovación es un motor decisivo del crecimiento y los "agentes" innovan para conservar una parte del mercado que de otra manera 
no retendrían, entonces la protección a la propiedad intelectual podría fomentar el crecimiento de largo plazo (Gould \& Gruben, 1996: 266).

Benavides \& Forero (2002) presentan un modelo de crecimiento endógeno y revisan los planteamientos de Lucas y Romer, quienes enfatizan en la importancia de la acumulación de capital humano o la variación del conocimiento tecnológico como fuentes de crecimiento autosostenido en el largo plazo. Benavides \& Forero (2002) demuestran que bajo ciertos supuestos, y en ausencia de externalidades, es posible obtener crecimiento sostenido, siempre que se permita el crecimiento del capital humano y de la tecnología. Pero en cualquier otro caso, el crecimiento del producto termina agotándose y el modelo se comporta de la misma manera que el de Solow (1957), por ello la protección de los DPI permite mayor acumulación de tecnología, debido a que el capital humano por sí solo no garantiza un crecimiento sostenido.

Según Benavides \& Forero (2002) "si el conocimiento tecnológico es un factor no rival, parcialmente excluible, que puede crecer, es necesario adoptar una estructura de mercado distinta de la competencia perfecta, puesto que esas características hacen necesario incentivos para invertir en su producción, que se pueden crear mediante estructuras monopólicas"( p. 126 ).

En consecuencia, la propensión de las empresas a apropiar dichos rendimientos está en función de la inversión en generación de nuevos conocimientos: "(...) es cierto que los agentes privados maximizadores de sus ganancias invierten en la creación de nuevo conocimiento, y obtienen un rendimiento sobre estas inversiones cobrando por los bienes resultantes un precio mayor que el costo marginal de su producción” (Romer 1990: 464).

Según Gould \& Gruben (1996) aunque un país esté dispuesto a adquirir nueva tecnología no lo logrará debido a que "Cuando se trata de negociar con empresas de países como éstos, los productores de tecnología extranjeros son muy cautelosos para venderla, en vista de que los compradores pueden violar impunemente los acuerdos de compra" (p. 270). Por tanto, de acuerdo con Gould \& Gruben (1996) una protección débil de los DPI en un país hace que las empresas que producen tecnología de punta no estén dispuestas a exportar sus productos a dicho país debido a que se incrementa la posibilidad de copia, lo que prolonga el rezago tecnológico de los países demandantes de tecnología.

Para Gould \& Gruben (1996) el comercio abierto implica que las empresas locales probablemente enfrenten la competencia de productores extranjeros que usan la tecnología más avanzada tanto en sus procesos de producción como en sus productos. Las empresas locales que desean hacer frente a este reto mediante la compra de tecnología extranjera pueden descubrir que una protección débil de la propiedad intelectual en su país de origen es un obstáculo para sus esfuerzos.

El modelo de Iwaisako \& Futagami (2013) construido con base en el modelo de crecimiento con una expansión de variedad de productos de Grossman \& Helpman (1991, cap. 3), el cual incluye la acumulación de capital, indaga cómo el fortalecimiento de la protección de patentes afecta el crecimiento económico. En dicho modelo, aplicado en una economía cerrada, una protección más fuerte de patentes plantea beneficios para la innovación, pero reduce la demanda de factores de capital, lo cual puede impedir el crecimiento económico. Los autores plantean que en un modelo de economía abierta en la que se transfieren tecnologías y el capital se importa del extranjero, una protección estricta de las patentes aumenta la adopción de la tecnología del extranjero, pero impide la acumulación de capital, estableciendo que la relación derivada de la protección de patentes no es monótona (Iwaisako \& Futagami, 2013: 631,).

En el modelo de Iwaisako \& Futagami (2013), el volumen total de las actividades de I + D en la economía determina la tasa de crecimiento del número de bienes diferenciados, y esto es un indicador del crecimiento de la productividad de la economía. Los partidarios de la aplicación de un sistema leve de DPI critican la defensa teórica que propenden los modelos de crecimiento actuales. Según Vercello- 
ne (2004: 69) "La ampliación y la prolongación en el tiempo de los derechos de propiedad intelectual serían ahora la condición esencial de la innovación, permitiendo a las firmas amortizar sus costes en $I+D$. Hay que destacar que esta defensa teórica del papel de las patentes, situada en el corazón de las nuevas teorías del crecimiento endógeno, resulta falaz en bastantes aspectos. Se revela como un instrumento ideológico que justifica los nuevos cercamientos del saber y la exclusión del Sur del acceso a la nueva división cognitiva del trabajo".

Existe la posibilidad de innovación y crecimiento en ausencia de DPI, por ejemplo Cozzi (2009) sostiene que como el motor del crecimiento económico es la innovación, entonces los DPI no son necesariamente fundamentales para fomentar la innovación y el crecimiento. Para Acevedo et al. (2012: 47) el régimen de DPI permite a los innovadores ser recompensados por sus innovaciones, entonces la innovación sería posible en ausencia de DPI a través de otros medios, como la educación.

Los nuevos productos y procesos que serán usados por las empresas e intercambiados en el comercio internacional, por ende, estarán determinados por el régimen de DPI que esperan las empresas del país oferente de tecnología de punta, esto de alguna manera genera restricciones al uso del conocimiento y desestimula la competencia y la innovación en el país receptor. Para Caviedes \& Fuentes (2011: 147) las empresas grandes que han comenzado actividades en I+D, usando el sistema de DPI, solicitan tantas patentes como les sea posible en un campo tecnológico, con el objetivo de ocupar esta área del conocimiento y desestimular a la competencia para que incursione en este campo; además, conducen a la formación de monopolios, restringiendo el acceso al conocimiento y no son un instrumento para impulsar la investigación al servicio de la sociedad.

Según Claire Lelarge (2012), se puede presentar un estancamiento en el proceso innovador en un país receptor de tecnología que a su vez fortalece su sistema de protección de DPI dado que aunque las patentes proporcionan incentivos y pueden facilitar la difusión de tecnología y la creación de empresas y mercados para la tecnología; sin embargo, también se pueden utilizar a manera de evitar la competencia, crear distorsiones de monopolios así como el impedimento de innovaciones subsiguientes. Bajo este mismo argumento Lerner (2000) analiza los cambios en la política de patentes en 60 países entre 1850 y el año 2000, encontrando que cuando un país fortalece su sistema de patentes, recibe más inventos de otros países. Sin embargo, los inventores nacionales no parecen patentar más sus inventos ni en su país ni en el extranjero, lo que sugiere que no existe un impacto significativo en la innovación nacional (Lelarge, 2012: 203).

Caviedes \& Fuentes (2011), advierten que existen desventajas en materia de negociación en los términos de protección de DPI a raíz de los acuerdos de integración regional o ADPIC y Adpic- Plus, y estas se relacionan con las diferencias subyacentes en las estructuras productivas entre países:

"El régimen de Propiedad Intelectual (PI) a nivel mundial se desarrolla bajo el supuesto de que los sujetos económicos pueden actuar bajo iguales condiciones, desconociendo por completo las asimetrías que existen entre países desarrollados y subdesarrollados, o en vías de desarrollo" (p. 145); además encuentran que dichos acuerdos trajeron consigo mayores restricciones para la PI como “... la extensión en tiempo de la cobertura, ampliación de la materia susceptible a ser protegida por la legislación y mayor grado de vigilancia con los temas relacionados; además se obliga a los países firmantes a restringir el otorgamiento de licencias obligatorias para casos de fuerza mayor o por conveniencia nacional. Se niegan las importaciones paralelas, políticas estas que, en algún momento, fueron usadas por los países desarrollados" (Caviedes \& Fuentes, 2011: 159-160).

Los críticos a la protección fuerte de los DPI también argumentan que un régimen fuerte limita la diseminación de nuevas ideas dadas las restricciones de acceso, manifiestan que proteger la información 
científica interrumpe los procesos de innovación, así mismo establecen que solo una protección más débil permite obtener las externalidades positivas y realizar los spillovers, puesto que al copiar la tecnología de punta de los países productores la innovación surge en un ambiente de acceso libre al conocimiento ${ }^{7}$. La imitación ha sido una forma comúnmente usada por los países en desarrollo para intentar aproximarse al nivel tecnológico de los países desarrollados. La copia de las técnicas por parte de los países tecnológicamente rezagados propicia la innovación. Por tanto, un régimen de DPI fuerte solamente beneficia a las empresas productoras y poseedoras de las patentes y del conocimiento de punta en lugar de estimular la innovación por copia de productos y procesos novedosos del país receptor de bienes de mayor conocimiento incorporado y valor agregado.

Como se observa, la protección de los DPI proporciona a las empresas los incentivos que necesitan para la invertir en investigación y hacer uso del conocimiento generado, pero la aplicación de medidas fuertes de DPI también se pueden utilizar para evitar la competencia, creando distorsiones en los mercados como los monopolios, impidiendo además el uso por parte de las empresas y de la sociedad de un producto o innovación ${ }^{8}$, aunque la capacidad de copiar no desaparece. Cuando el crecimiento es impulsado por la innovación endógena, dos obstáculos se interponen en el camino de

7 Existen mecanismos de acceso libre al conocimiento implantados recientemente como el copyleft, el cual es una alternativa frente al derecho de autor, consiste en permitir la libre distribución de copias y versiones modificadas de una obra o trabajo que posea tal categorización; el Acceso abierto (Open Access), es el acceso inmediato sin requerimientos de registro, suscripción o pago a material digital educativo, académico, científico o de cualquier otro tipo $\mathrm{y}$, el software libre que junto con el copyleft se desarrolló para restringir las posibilidades de apropiación del software por parte de los usuarios y crear un sistema operativo construido con el apoyo mismo de los mismos.

8 EL caso de la farmacéutica Turing, los pacientes con sida dependen del medicamento que hasta hace poco costaba alrededor de $\$ 13.50$ la dosis. Pero Shkreli, el director ejecutivo de 32 años, anunció que estaría cobrando US\$750 por pastilla. Eso es más de $5.000 \%$ de aumento, arguyendo que las ganancias del medicamento crearían un mejor producto (BBC, 2015). la eficiencia del mercado. En primer lugar, la innovación requiere la existencia de beneficios de monopolio. En segundo lugar, la eficiencia exige que los retornos de inversión sean plenamente apropiables, pero las características del conocimiento sugieren que los spillovers serán frecuentes (Grossman \& Helpman, 1993: 37).

Teniendo en cuenta la importancia que adquieren los DPI para impulsar o detener la innovación y el cambio técnico al igual que sobre el crecimiento económico, se debe buscar un equilibrio entre la ganancia privada y el bienestar público para la distribución de los beneficios privados y sociales dadas las posiciones al respecto Vallejo (2017), Casas \& Barichelo (2105), especialmente en el marco de los acuerdos de integración económica y geopolítica que rigen en la actualidad. Por ejemplo, dentro de los ADPIC y ADPIC- Plus (Acuerdo de la OMC sobre los aspectos de los derechos de propiedad intelectual relacionados con el comercio), dichos acuerdos contienen elementos que tienen que ver con la forma como puede asumirse el proceso de crecimiento en los países, y que han sido ampliamente cuestionados por autores como Richardson \& Gaisford (1996), Roffe \& Santacruz (2006), Chomsky (2000), Zerda (2005a, 2005b, 2005c), entre otros.

\section{Análisis Econométrico}

El análisis de los efectos que tienen los DPI sobre el crecimiento económico se realizó tomando un conjunto de 66 países $^{9}$ de diferentes niveles de ingre-

\footnotetext{
9 Los países son: Ingreso Alto: OECD (6): Uruguay, Argentina, Singapur, Qatar, Hong Kong, Federación De Rusia; Ingreso Alto: OECD (26): Suiza, Suecia, Australia, Austria, Bélgica, República Checa, Reino Unido, Portugal, Nueva Zelandia, Noruega, Canadá, Chile, Luxemburgo, Japón, República De Corea, Italia, Israel, Dinamarca, Islandia, Irlanda, Hungría, Estados Unidos, Estonia, Grecia, Francia, Finlandia; Ingreso medio alto (17): Albania, Perú, México, Libia, Kazakstán, Jordán, Bosnia Y Herzegovina, Botswana, Brasil, Bulgaria, Jamaica, Irán, China, Colombia, Ecuador, Costa Rica y Cuba; Ingreso medio bajo (12): Nigeria, Nicaragua, Kenia, Indonesia, India, Bolivia, Honduras, Guyana, Guatemala, Ghana, España, Egipto; Ingreso bajo (4): República Dominicana, Nepal, Haití, Etiopia.
} 
so ${ }^{10}$ entre 1990 a 2014 , y se buscó comprender la incidencia de proteger los DPI sobre la producción del país; por tanto, el modelo utiliza una formulación log-lineal al igual que el modelo base de Gould \& Gruben (1996), el cual se basa en una función de producción de Cobb-Douglas aumentada a DPI. Entonces, el modelo a estimar es:

$$
\begin{aligned}
\text { CPIBPc }_{i}= & \beta_{0} \\
+ & \beta_{1} \text { fbk }_{i}+\beta_{2} \text { educ }_{i}+\beta_{3} \text { ID }_{i}+\beta_{5} \text { Lipri }_{i}+\beta_{6} \text { LPIBpc }_{i} \\
& +\beta_{4} \text { gppib }_{i}+\beta_{7} \text { gapert }_{i}+\beta_{8} \text { ralta }_{i}+\beta_{9} \text { rbaja }_{i}+\mu_{i}
\end{aligned}
$$

Para el ejercicio econométrico se tomaron los promedios de los datos del Crecimiento del PIB per cápita $\left(C P I B p c_{i}\right)$, teniendo en cuenta el criterio del Banco Mundial para definir a los países $i$ de renta alta, media y baja. Las variables explicativas son: la formación bruta de capital respecto al PIB $f b k_{i}$, la cantidad de personas con educación terciaria en cada país como proporción de la población total $\left(e d u c_{i}\right)$, el gasto en I+D respecto al PIB $\left(I D_{i}\right)$, el logaritmo natural del índice IPRI como proxy del esfuerzo de protección con DPI $\left(\right.$ Lipri $\left._{i}\right)$, el logaritmo natural del PIB per cápita de 1990 LPIBpc $_{i}$ ), que permite establecer la existencia de convergencia entre países. Finalmente, como variables control se toman: el gasto público respecto al PIB $\left(g p p i b_{i}\right)$, el grado de apertura económica (gapert $_{i}$ ) y las dummys que identifican los países según su renta.

El IPRI (International Property Rights Index) ${ }^{11}$ o Índice internacional de propiedad intelectual, fue desarrollado para medir la situación de los derechos de propiedad en el mundo, tiene tres componentes básicos: Ambiente Legal y Político, es el impacto significativo en el desarrollo y la protección de los derechos de propiedad física e intelectual, muestra de la estabilidad y la independencia política, legal y judicial de un país, el estado de derecho y el control de la corrupción; Derechos de Propiedad Físico, concierne la capacidad de protección de los derechos de propiedad física de los agentes, el registro de la propiedad y acceso a créditos; y finalmente Derechos de Propiedad Intelectual, lo cual refleja la protección y el control a las patentes y los derechos de autor (Alliance, 2014: 5). La clasificación del IPRI utiliza una escala de 0 a 10 , donde 10 es el valor más alto para un sistema de derechos de propiedad fuerte y 0 es el valor más bajo para un sistema de protección derechos de propiedad débil dentro de un país. Los resultados del análisis IPRI sugieren que las economías más desarrolladas se caracterizan por un alto IPRI, lo que implica que existe una relación positiva entre el desarrollo económico y la fuerza de los regímenes de derechos de propiedad (Alliance, 2014: 4).

Los datos se obtuvieron de diversas fuentes como: Las bases de datos del Banco de Mundial, la organización mundial de la propiedad intelectual OMPI y la alianza por los derechos de propiedad (PRA).

10 Para fines operativos y analíticos, las economías se dividen entre los grupos de ingresos de acuerdo al (INB) ingreso nacional bruto per cápita 2014, calculado utilizando el método Atlas del Banco Mundial. Los grupos son: renta baja, \$ 1,045 o menos; renta media baja, \$ 1,046-4,125; renta media alta, \$ 4,126-12,735; y renta alta, \$12,736 o más (Banco Mundial, s.f.).

11 El Índice Internacional de Derechos de Propiedad (IPRI) es la principal publicación de la Alianza por los Derechos de Propiedad (PRA) en Washington. Es un estudio comparativo anual que tiene como objetivo cuantificar la fuerza de los derechos de propiedad - tanto física como intelectual. El (IPRI) sirve como un indicador para el estado del desarrollo de derechos de propiedad física e intelectual en todo el mundo. También ha puesto de manifiesto la importante relación

entre los derechos de propiedad, la libertad económica y el nivel de desarrollo de un país. (Alliance, 2014). 
Tabla 2. Descripción de variables para el modelo propuesto

\begin{tabular}{|c|c|c|c|c|}
\hline Variable & Abreviación & Medido en & Descripción & Fuente \\
\hline $\begin{array}{l}\text { Crecimiento del } \\
\text { PIB per cápita }\end{array}$ & CPIBpc $c_{i}$ & $\begin{array}{l}\text { Tasa de variación } \\
\text { promedio entre } \\
\text { 1990-2014 }\end{array}$ & $\begin{array}{l}\text { La tasa de variación promedio del PIB per-cápita entre } \\
1990-2004 \text { es la explicación del avance en el desa- } \\
\text { rrollo económico de un país. }\end{array}$ & $\begin{array}{l}\text { Banco Mundial y archivos de } \\
\text { datos sobre cuentas naciona- } \\
\text { les de la OCDE (Organización } \\
\text { para la Cooperación y el De- } \\
\text { sarrollo Económico) }\end{array}$ \\
\hline $\begin{array}{l}\text { Formación bruta de } \\
\text { Capital }\end{array}$ & $f b k_{i}$ & $\%$ del PIB & $\begin{array}{l}\text { La formación bruta de capital (anteriormente, inver- } \\
\text { sión interna bruta) comprende los desembolsos en } \\
\text { concepto de adiciones a los activos fijos de la eco- } \\
\text { nomía más las variaciones netas en el nivel de los } \\
\text { inventarios. Refleja la tasa de ahorro en el estado } \\
\text { estacionario. }\end{array}$ & $\begin{array}{l}\text { Banco Mundial y archivos de } \\
\text { datos sobre cuentas naciona- } \\
\text { les de la OCDE }\end{array}$ \\
\hline $\begin{array}{l}\text { Población activa } \\
\text { con educación } \\
\text { terciaria }\end{array}$ & $e d u c_{i}$ & $\begin{array}{l}\text { \% Total de la } \\
\text { población }\end{array}$ & $\begin{array}{l}\text { Proporción de la población activa que posee una } \\
\text { educación terciaria, como porcentaje de la población } \\
\text { activa total. }\end{array}$ & $\begin{array}{l}\text { Banco Mmundial, base de da- } \\
\text { tos de Indicadores principales } \\
\text { sobre el mercado laboral. }\end{array}$ \\
\hline $\begin{array}{l}\text { Gasto en } \\
\text { Investigación y } \\
\text { Desarrollo }\end{array}$ & $I D_{i}$ & $\%$ del PIB & $\begin{array}{l}\text { Los gastos en investigación y desarrollo son gastos } \\
\text { corrientes y de capital (público y privado) en trabajo } \\
\text { creativo realizado para incrementar los conocimien- } \\
\text { tos, incluso los conocimientos sobre la humanidad, } \\
\text { la cultura y la sociedad, y el uso de los conocimientos } \\
\text { para nuevas aplicaciones. }\end{array}$ & $\begin{array}{l}\text { Banco Mundial y archivos de } \\
\text { datos sobre cuentas naciona- } \\
\text { les de la OCDE }\end{array}$ \\
\hline Gasto Público & gppib $_{i}$ & $\%$ del PIB & $\begin{array}{l}\text { Los gastos son los pagos de dinero por actividades } \\
\text { operativas del Gobierno para la provisión de bienes } \\
\text { y servicios. Incluye remuneración de empleados } \\
\text { (como sueldos y salarios), interés y subsidios, do- } \\
\text { naciones, beneficios sociales y otros gastos como } \\
\text { renta y dividendos. }\end{array}$ & $\begin{array}{l}\text { Se construye de las estadís- } \\
\text { ticas del Banco Mundial y ar- } \\
\text { chivos de datos sobre cuen- } \\
\text { tas nacionales de la OCDE }\end{array}$ \\
\hline $\begin{array}{l}\text { Índice Internacional } \\
\text { de Derechos de } \\
\text { Propiedad }\end{array}$ & Lipri $_{i}$ & $0-10$ & $\begin{array}{l}\text { El IPRI (International Property Rights Index) o Índice } \\
\text { internacional de propiedad Intelectual, fue desarrolla- } \\
\text { do para medir la situación de los derechos de propie- } \\
\text { dad en el mundo }\end{array}$ & Alliance (2014). \\
\hline PIB Per cápita & $L P I B p c_{i}$ & $\begin{array}{l}\text { Logaritmo natural } \\
\text { del PIB per-cápita } \\
\text { de } 1990\end{array}$ & $\begin{array}{l}\text { Es la variable que permite identificar el desarrollo en } \\
\text { el punto de partida del análisis }\end{array}$ & $\begin{array}{l}\text { Se construye de las estadís- } \\
\text { ticas del Banco Mundial y ar- } \\
\text { chivos de datos sobre cuen- } \\
\text { tas nacionales de la OCDE }\end{array}$ \\
\hline $\begin{array}{l}\text { Grado de apertura } \\
\text { comercial }\end{array}$ & gapert $_{i}$ & $(\mathrm{M}+\mathrm{X}) / \mathrm{PIB}$ & $\begin{array}{l}\text { Participación del comercio internacional de un país } \\
\text { respecto al PIB }\end{array}$ & $\begin{array}{l}\text { Se construye de las estadís- } \\
\text { ticas del Banco Mundial y ar- } \\
\text { chivos de datos sobre cuen- } \\
\text { tas nacionales de la OCDE }\end{array}$ \\
\hline Países de renta alta & ralta $_{i}$ & $\begin{array}{l}\text { Dummy que } \\
\text { señala } 1 \text { si es país } \\
\text { de renta alta y } 0 \text { si } \\
\text { no lo es }\end{array}$ & $\begin{array}{l}\text { Identifica las diferencias de los países de acuerdo al } \\
\text { nivel de ingresos }\end{array}$ & Clasificación Banco Mundial \\
\hline $\begin{array}{l}\text { Países de renta } \\
\text { baja }\end{array}$ & rbaja $_{i}$ & $\begin{array}{l}\text { Dummy que } \\
\text { señala } 1 \text { si es país } \\
\text { de renta baja y } 0 \text { si } \\
\text { no lo es }\end{array}$ & $\begin{array}{l}\text { Identifica las diferencias de los países de acuerdo al } \\
\text { nivel de ingresos }\end{array}$ & Clasificación Banco Mundial \\
\hline
\end{tabular}

Fuente: elaboración de los autores. 
La estimación se realiza partiendo de datos panel para llegar a datos en corte transversal, lo cual lleva a obtener estimadores de efectos fijos entre grupos (between), que identifican efectos de largo plazo y permiten eliminar la heterogeneidad no observada. Este método lleva a que las variables estacionarias se encuentren distribuidas alrededor de su valor de largo plazo, y permite eliminar el efecto de los ciclos en las variables (Rosales et al, 2010). El IPRI se toma en ausencia de una estadística de DPI, siendo relevante tomar una proxy que permita evitar los errores en la estimación que conduzca a un sesgo en el resultado del estimador.

Tabla 3. Efecto de los DPI sobre el crecimiento económico

\begin{tabular}{|c|c|c|c|}
\hline \multicolumn{4}{|c|}{ Variable Dependiente: Crecimiento del PIB per cápita $\left(C P I B p c_{i}\right)$} \\
\hline \multicolumn{4}{|l|}{ Muestra: 65 países } \\
\hline \multicolumn{4}{|l|}{ Mínimos Cuadrados Ordinarios (MCO) } \\
\hline \multicolumn{4}{|c|}{ Errores estándar consistentes con heterocedasticidad } \\
\hline \multicolumn{4}{|l|}{ Errores estándar ( ) } \\
\hline Independientes & [1] & [2] & [3] \\
\hline \multirow[t]{2}{*}{ Constante } & 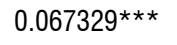 & $0.09818^{\star * \star}$ & $0.071828 * \star \star$ \\
\hline & $(0.016683)$ & $(0.019703)$ & $(0.02076)$ \\
\hline \multirow[t]{2}{*}{ FBK respecto al PIB $\left(f b k_{i}\right)$} & $0.090766^{\star}$ & 0.056682 & $0.060459 * *$ \\
\hline & $(0.050635)$ & $(0.041366)$ & $(0.029724)$ \\
\hline \multirow[t]{2}{*}{ Educación terciaria $\left(e d u c_{i}\right)$} & 0.004246 & -0.005179 & 0.002797 \\
\hline & $(0.011855)$ & $(0.012265)$ & $(0.013098)$ \\
\hline \multirow[t]{2}{*}{$\mathrm{I}+\mathrm{D}$ respecto al $\mathrm{PIB}\left(I D_{i}\right)$} & & $0.593154^{\star \star \star}$ & $0.426196^{\star \star}$ \\
\hline & & $(0.209913)$ & $(0.199862)$ \\
\hline \multirow[t]{2}{*}{ Índice propiedad intelectual $\left(\right.$ Lipri $\left._{i}\right)$} & & & $0.030914^{\star * *}$ \\
\hline & & & $(0.01057)$ \\
\hline \multirow[t]{2}{*}{ PIB per-cápita $\left(L P I B p c_{i}\right)$} & $-0.007396^{\star \star \star}$ & $-0.009936^{\star \star \star}$ & $-0.012509 * \star *$ \\
\hline & $(0.002181)$ & $(0.002351)$ & $(0.002235)$ \\
\hline \multirow[t]{2}{*}{ Gasto público respecto al PIB $\left(g p p i b_{i}\right)$} & -0.035729 & $-0.064298^{\star *}$ & $-0.100098^{* * *}$ \\
\hline & $(0.024325)$ & $(0.02563)$ & $(0.030409)$ \\
\hline \multirow[t]{2}{*}{ Grado de apertura comercial $\left(\right.$ gapert $\left._{i}\right)$} & $0.003096 *$ & $0.004362 * * *$ & 0.00232 \\
\hline & $(0.001617)$ & $(0.001557)$ & $(0.002061)$ \\
\hline \multirow[t]{2}{*}{ Países renta baja $\left(r b a j a_{i}\right)$} & $-0.014089 * *$ & $-0.018717^{\star \star \star}$ & $-0.019089 * * *$ \\
\hline & $(0.005387)$ & $(0.005502)$ & $(0.00452)$ \\
\hline
\end{tabular}




\begin{tabular}{|l|c|c|c|}
\hline & & & 0.002984 \\
\hline Países renta alta $\left(\right.$ ralta $\left._{i}\right)$ & 0.005131 & $(0.004664)$ & 0.002505 \\
\hline R-cuadrado & $(0.004853)$ & 0.608215 & $0.004372)$ \\
\hline Errores estándar regresión & 0.541736 & 0.009151 & 0.008438 \\
\hline Criterio de Akaike & 0.477579 & -6.408182 & -6.554101 \\
\hline Observaciones & -6.285931 & 65 & 65 \\
\hline
\end{tabular}

*** Significativo al 1\%, ${ }^{* *}$ significativo al $5 \%, y$ * significativo al $10 \%$

Fuente: cálculos propios

En el tabla 3 se muestran los resultados de las estimaciones econométricas, en donde se consideran tres diferentes modelos: el primero asume que el crecimiento económico es explicado por los factores tradicionales (capital, educación y los controles por países), el segundo refleja el papel que tiene el gasto en $\mathrm{I}+\mathrm{D}$ respecto al $\mathrm{PIB}$, y en el tercero se muestra la influencia de complementar los efectos que tiene el gasto en I+D con DPI. Además, según los estadísticos para la selección del mejor modelo, indican que la regresión [3] tiene mayor poder de predicción (mayor $\mathrm{R}$ cuadrado respecto a [1] y [2]), los errores estándar de la regresión son los menores y lo valida el criterio de selección de Akaike.

En los resultados de los modelos [2] y [3] se refleja que el gasto en I+D es importante para el crecimiento económico, dado que cuando los países elevan en $1 \%$ este gasto respecto al PIB el crecimiento económico per cápita es $0.43 \%$ más alto, siendo coherente con el resultado de la propuesta de Ginarte \& Park (1997), quienes evidencian que altos niveles de gasto en actividades de I+D es lo que caracteriza a las economías innovadoras. Sin embargo, resaltan que: "no es el stock de capital humano per se lo que genera incentivos para proporcionar protección de patentes, sino más bien las inversiones o recursos comprometidos con la creación de conocimiento" (Ginarte \& Park, 1997: 299).

El análisis empírico de Ginarte \& Park (1997) considera que la libertad de mercado es un fuerte de- terminante de los niveles de protección de patentes para todos los países, y que las tasas de inversión en $\mathrm{I}+\mathrm{D}$ son un factor determinante para la protección de las patentes en las economías desarrolladas. Además, sugieren que si el tamaño del sector de la investigación es pequeño, los inventores pueden que no produzcan las suficientes innovaciones para la adopción de un sistema de patentes que valga la pena; en cambio, los países que llevan a cabo investigaciones innovadoras significativas son más propensos a tener intereses creados en ver respetados sus derechos de patente (Ginarte \& Park, 1997).

Al respecto Gould \& Gruben (1996) sugieren que el país consumidor neto de innovaciones sólo se sentirá motivado a proteger la propiedad intelectual cuando el tipo de innovación que demande sea diferente del demandado en el país productor neto de innovaciones, de lo contrario no le favorecería un régimen fuerte, complementan lo anterior diciendo que: "(...) Si el país consumidor demanda innovaciones que son muy diferentes de las producidas por el país innovador, este último aun innovará en beneficio del país consumidor, si éste protege la propiedad intelectual" (Gould \& Gruben, 1996).

Respecto a la $f b k_{i}$ se puede evidenciar que es estadísticamente significativa al momento de explicar el crecimiento económico, donde un aumento de $1 \%$ en la variación de la relación capital-producto lleva a que el crecimiento per cápita sea $0.06 \%$ más alto, lo cual refleja la importancia de la acumulación de capital físico para la expansión de la economía. 
Las regresiones evidencian que, estadísticamente, el efecto de la inversión en capital físico es altamente significativo cuando se adiciona LIPRI, demostrando que en los países existe una complementariedad entre acumular capital físico y fortalecer la definición de los derechos de propiedad intelectual. Esto no significa que se esté mostrando una causalidad entre las dos variables, sino que son dos condiciones importantes para que los países avancen en su desarrollo económico.

En la estimación se puede demostrar la convergencia condicional de los países (en los tres modelos el resultado del efecto de $L P I B P c$ es estadísticamente significativo y con signo negativo), ya que situándose en el estado estacionario los países con tasas de ahorro similares pueden divergir y países con diferentes tasas de ahorro pueden llegar a converger; por tanto, uno de los aspectos que llevan a que los países tiendan a ser similares es la inversión en conocimiento (gasto en I+D) y la fortaleza de la protección intelectual (obsérvese en el cuadro 3 que la inclusión de los DPI y el gasto en I+D eleva el valor del beta de convergencia). Ahora, la no significancia de la variable ocurre porque los efectos del conocimiento vienen inmersos en y en ID, y Lipri, además, en el marco temporal de análisis tiende a existir una convergencia de los países hacia niveles similares de personas con educación terciaria respecto al total, donde esta formación no necesariamente se traduce en nuevo conocimiento para impulsar la diferenciación de la productividad.

Analizando las diferentes variables control se puede denotar que una mayor participación del gasto público dentro del PIB (gppib) va en contra del crecimiento, por lo que es posible deducir una relación entre los problemas de déficit de los gobiernos y el crecimiento de largo plazo; según Posada (2015) el incremento del gasto público en Colombia entre 1970 y 2000 redujo la eficiencia del proceso de innovación, que en definitiva se tradujo en un menor cambio técnico.

De otro lado, se estima que el grado de apertura (gapert) no explica significativamente al crecimiento cuando se adicionan los DPI, pero cuando no son incluidos el comercio internacional tiene un grado de incidencia sobre el crecimiento de largo plazo, resultado que se sustenta cuando países más innovadores tienden a ser exportadores más competitivos. Finalmente, la diferencia en el crecimiento del PIB per cápita según la renta de los países es estadísticamente significativa para los países de renta alta y media respecto a los de renta baja, donde en promedio estos últimos crecen $1.9 \%$ menos que los primeros.

Es evidente que la protección de la propiedad intelectual, medida a través del índice IPRI, es importante para explicar el crecimiento del PIB per cápita de los países, debido a la importancia que adquieren cuando se protegen las innovaciones $y$, de esa manera, se generan incentivos económicos que inducen a nuevas dinámicas de inversión; por tanto, los países cuyas empresas hacen uso intensivo del conocimiento no se beneficiarán de un escenario de protección débil de los DPI, debido a la posibilidad de imitación -learning by doing- y la capacidad de apropiación de las rentas. Por tanto, los resultados se encuentran alineados con los obtenidos por Alliance (2014), donde se comprueba la relación positiva entre el Ingreso de los Hogares, el Producto Interno Bruto Per Cápita y la Inversión Extranjera Directa con la puntuación IPRI de los países.

\section{Conclusiones}

Los resultados del modelo estimado muestran que el gasto en I+D es importante para el crecimiento económico, lo cual es coherente con la teoría propuesta por Ginarte \& Park (1997) quienes encuentran que un alto nivel de capital humano y la I+D son actividades que caracterizan las economías innovadoras. Analizando los datos estadísticos y observando los resultados del ejercicio econométrico es posible observar cómo un alto nivel de inversión en $\mathrm{I}+\mathrm{D}$ y un alto índice IPRI son elementos que caracterizan las economías innovadoras y competitivas; por tanto, existe una menor tendencia a proteger los DPI en los países con capacidades innovadoras inferiores o menores niveles de desarrollo tecnológico.

La adopción del sistema de patentes depende del tamaño del sector de la investigación de un país, uno pequeño no produce las suficientes innova- 
ciones para la adopción de un sistema de patentes fuerte, pero las naciones con derechos de patentes más débiles deben fortalecer sus regímenes a cambio de la colaboración o asistencia en investigación, lo cual es algo contradictorio, debido a que la imitación es una forma que ha permitido el desarrollo tecnológico para los países rezagados. Entonces, proporcionar una mayor regulación para los DPI de empresas extranjeras podría paralizar los proyectos de tecnología.

Por su parte, las relaciones entre las variables I+D y el PIB per cápita indican que los DPI seguirán siendo considerados como un elemento importante para analizar el desempeño tecnológico de un país y, por ende, el crecimiento económico (especialmente los países desarrollados con tecnología de punta). En ese sentido, se supondría que mediante la implementación adecuada de una política de protección y cesión de DPI, enfocada al impulso y desarrollo del cambio tecnológico, puede ser posible alcanzar un equilibrio entre los intereses públicos y privados en el uso del conocimiento, para que se aprovechen las ventajas del mismo sobre la innovación y el cambio tecnológico que propenda hacia un incremento de la productividad en cada sector de la economía y, por ende, en el bienestar social.

Finalmente, se debe señalar que existen líneas de investigación abiertas asociadas a los elementos relativos a la producción y la protección del conocimiento a nivel microeconómico y el análisis de las maneras para conducir una mayor transferencia tecnológica entre países con altas tasas de acumulación de conocimiento. Además, en próximos estudios sería pertinente abordar el estudio sobre los costos de desarrollar y mantener sistemas de DPI en los países y la generación de spillovers.

\section{Referencias}

Alliance, P. R. (2014). International Property Rights Index. 2014 Report. Property Rights Alliance, Washington, DC. Recuperado de http://internationalpropertyrightsindex.org/about

Acevedo, M. L., Afonso, O., \& Silva, S. T. (2012). Intellectual Property Rights and Endogenous Economic Growth-Uncovering the Main Gaps in the Research Agenda. INTECH Open Access Publisher.
Banco Mundial (s.f) Estadisticas. Recuperado de https://datos. bancomundial.org/

Barro, R. J., \& I Martin, X. S. (2009). Crecimiento económico. Barcelona: Reverté.

BBC (2015) Martin Shkreli: ¿es éste el hombre más odiado de Estados Unidos? Recuperado de http://www.bbc.com/ mundo/noticias/2014/05/140502_finde_economia_costo_nuevas_drogas_aa.shtml

Benavides, G., Óscar, A. \& Forero, P. (2002). Crecimiento endógeno: conocimiento y patentes. Revista de Economía Institucional, 4(6), 109-131.

Casas-Herrera, J., \& Barichello, R. (2015). Hacia una noción sobre la pobreza. APUNTES DEL CENES, 34(59), 39-62. doi:https://doi.org/10.19053/22565779.2784

Caviedes. C., \& Fuentes. H. (2011). Acuerdos de propiedad intelectual: ¿Nos acercan al desarrollo?. Apuntes del CENES, 30(51), 141-164.

CECTE (2008). Ética en el acceso al conocimiento de dominio público. Recuperado de http:// www.mincyt.gob.ar/_post/ descargar.php?idAdjuntoArchivo $=44273$

Chen, Y. \& T. Puttitanun (2005). Intellectual property rights and innovation in developing countries. Journal of Development Economics, 78(2): 474-493. http://dx.doi.org/10.1016/j. jdeveco.2004.11.005

Chomsky, N. (2000). La sociedad global. Educación, mercado y democracia.

Corsani, A. (2004). Hacia una renovación de la economía política. Antiguas categorías e innovación tecnológica. En Blondeau, O., \& Sánchez Cedillo, R. (1 Ed) Capitalismo cognitivo: propiedad intelectual y creación colectiva. Madrid: Traficantes de Sue-os. (pp. 89 -98)

Cozzi, G. (2009). Intellectual Property, innovation, and growth: introduction to the special issue. Scottish Journal of Political Economy, 56(4), 383-389. http://dx.doi.org/10.1111/j.14679485.2009.00490.x

Gadbaw, R. M., \& Richards, T. (1988). Intellectual property rights. Global consensus, global conflict.

Ginarte, J.C. y W.G. Park (1997). Intellectual Property Rights and Economic Growth. Contemporary Economic Policy, $\mathrm{XV}: 51-61$.

Gould, D.M. \& Gruben, W.C., (1996). The role of intellectual property rights in economic growth. Journal of Development Economics 48: 323-350. http://dx.doi.org/10.1016/03043878(95)00039-9

Grossman, G. M., \& Helpman, E. (1993). Endogenous innovation in the theory of growth. National Bureau of Economic Research NBER Working Paper No. w4527. http://dx.doi. org/10.3386/w4527

Guzmán, A. (2000). Las fuentes endógenas del crecimiento económico. Economía Teoría y Práctica: 35-60. Recuperado de: http://www.izt.uam.mx/economiatyp/numeros/numeros/13/ articulos_PDF/13_2_Las_fuentes_endogenas.pdf

Lelarge, C. (2012). Innovación y los derechos de propiedad intelectual. En OCDE. Innovación en las empresas. Foro consultivo científico y tecnológico. (pp 201-245) Recupera- 
do de http://www.foroconsultivo.org.mx/libros_editados/ innovacion_empresas.pdf

Lerner, J. (2000). 150 years of patent protection. National bureau of economic research No. w7478.

Lucas, R. (1988). On the mechanics of Economic Development. Journal of Monetary Economics, 22(1): 3-42. http://dx.doi. org/10.1016/0304-3932(88)90168-7

Iwaisako, T., \& Futagami, K. (2013). Patent protection, capital accumulation, and economic growth. Economic Theory, 52(2), 631-668. http://dx.doi.org/10.1007/s00199-011-0658-y

Park, W. G. (2008). International patent protection: 1960-2005. Research policy, 37(4): 761-766. http://dx.doi.org/10.1016/j. respol.2008.01.006

Posada, C. E. (2015). El costo de oportunidad del cambio técnico, el crecimiento económico y el caso colombiano 1925-2012. Ensayos sobre Política Económica, 33(77), 149-167.

Rapp, R. T., \& Rozek, R. P. (1990). Benefits and costs of intellectual property protection in developing countries. Journal of world trade, 24(5): 75-102.

Rebelo, S. T. (1991). Long run policy analysis and long run growth. National Bureau of Economic Research NBER Working Paper No. 3325. http://dx.doi.org/10.1086/261764

Richardson, R. S., \& Gaisford, J. D. (1996). North-South disputes over the protection of intellectual property. The Canadian Journal of Economics/Revue canadienne d'Economique, 29, S376-S381. http://dx.doi.org/10.2307/136071

Roffe, P., \& Santacruz, M. (2006). Los derechos de propiedad intelectual en los acuerdos de libre comercio celebrados por países de América Latina con países desarrollados. CEPAL.

Romer, P. M. (1983). Dynamic competitive equilibria with externalities, increasing returns and unbounded growth. Doctoral dissertation, University of Chicago, Department of Economics.

Romer, P. M. (1987). Growth based on increasing returns due to specialization. The American Economic Review, 77(2): 56-62.

Romer, P. M. (1991). El cambio tecnológico endógeno. El trimestre económico, 441-480.
Romer, P. M. (1994). The origins of endogenous growth. The journal of economic perspectives, 8(1): 3-22. http://dx.doi. org/10.1257/jep.8.1.3

Rosales, R.A, J.A Perdomo, C.A Morales \& J. A Urrego (2010). Fundamentos de econometría intermedia: teoría y aplicaciones. Apuntes de clase CEDE. Universidad de los Andes.

Solow, R. (1957). Technical change and the aggregate production function. Review of Economics and Statistics, 39(3): 312320. http://dx.doi.org/10.2307/1926047

Taylor, M. S. (1994). TRIPS, trade, and growth. International Economic Review, 35(2): 361-381. http://dx.doi. org/10.2307/2527058

Vercellone, C. (2004). Las políticas de desarrollo en tiempos del capitalismo cognitivo. En Emanuel Rodríguez y Raúl Sánchez, (Compiladores.), Capitalismo cognitivo, propiedad intelectual y creación colectiva (pp. 63-74). Traficantes de Sue-os.

Sala-i-Martin, X. (2000). Apuntes de crecimiento económico. Antoni Bosch Editor.

Vallejo-Zamudio, L. (2017). El incierto crecimiento económico colombiano. APUNTES DEL CENES, 36(64), 9-10. doi:https:// doi.org/10.19053/01203053.v36.n64.2017.6511

Young, A. A. (1928). Increasing returns and economic progress. The economic journal, 38(152): 527-542. http://dx.doi. org/10.2307/2224097

Zerda, A. (2005a). Ética y conocimiento. El caso de la negociación del TLC Colombia - USA. Cuadernos Del Doctorado. Ética, Salud Y Vida. En:Colombia ISBN: 958-701-540-1 ed: Universidad Nacional de Colombia, v.1, p.23 - 34 Recuperado de http://www.doctoradosaludp.unal.edu.co/images/ cuadernos/cuaderno1.pdf

Zerda, A. (2005b). Impacto de las provisiones TRIPS-Plus contempladas en el TLC Colombia-Estados Unidos. Serie LATN Papers (Latin American Trade Network), (41).

Zerda, Á., Cortés, M., De la Hoz, G., Muriel, D., Marcela, D., \& Forero, D. (2005c). Impactos del Tratado de Libre Comercio Colombia-Estados Unidos en el sector salud del distrito capital. Investig. segur. soc. salud, (7), 69-94 